\title{
Singlicate analysis: should this be the default for biomarker measurements using ligand-binding assays?
}

\author{
Zhuqiu Ye*,1, Jing Tu ${ }^{1}$, Krishna Midde ${ }^{1}$, Mike Edwards $^{1}$ \& Patrick Bennett ${ }^{1}$ \\ ${ }^{1}$ Biomarker Services, PPD Laboratories, 2244 Dabney Road, Richmond, VA 23230, USA \\ *Author for correspondence: Tel.. +1 804977 8334; zhuqiu.ye@ppdi.com
}

First draft submitted: 12 March 2018; Accepted for publication: 27 March 2018; Published online: 20 June 2018

Keywords: biomarker $\bullet$ fit-for-purpose $\bullet$ ligand-binding assay $\bullet$ nonregulated bioanalysis $\bullet$ regulated bioanalysis $\bullet$ singlicate analysis

\section{Evolution of biomarker ligand-binding assays: past \& present}

Regulated ligand-binding assays (LBAs) must undergo stringent validation in order to provide sufficient confidence in the data. Enormous amount of time, expense and effort are consumed to develop a new drug and get it released for use. If the drug candidate meets with late stage failure, the losses can be disastrous. Therefore, pharmaceutical companies are constantly seeking ways to improve effectiveness in the drug development process with reduced costs and time. Advances in our understanding of biology and disease mechanisms have led to a rapidly growing interest in using biomarkers to enhance the drug discovery/development process. Traditionally, biomarkers have been widely analyzed in clinical laboratories for disease diagnosis, prognosis and strategizing therapeutic interventions. Biomarkers have also been used in later-stage clinical studies as an effective adjunct to assess drug efficacy and toxicity. In recent years, exploratory biomarkers have been increasingly utilized to help pharmaceutical companies make internal decisions on early stage clinical studies such as proof of concept, go-no-go decision and mechanism of action by offering rapid turnaround and fit-for-purpose method validation strategies in nonregulated environments [1].

Biomarker method validation typically evaluates fundamental parameters, including precision, accuracy, selectivity, specificity, parallelism and stability. Parallelism is critical to assess relative accuracy, sensitivity, matrix effect and minimum required dilution [2]. Precision and accuracy are essential to define assay reproducibility. There are many sources of assay variability, either within a run or between different runs. For example, traditional plate-based ELISA involves manual steps that tend to introduce human errors compromising either intra-assay or inter-assay precision and accuracy. For LBA in bioanalysis, it is a long-standing practice to perform duplicate (or even triplicate) analysis of calibration curves, QCs and samples to reduce potential assay variability.

However, in the modern LBA world, duplicate analysis may no longer serve its intended purpose. First, it is questionable if duplicate analysis improves assay variability. Conventional 'duplicate analysis' is performed by pipetting a sample into two adjacent wells. Today's handheld pipettes are manufactured to be highly repeatable. Use of automated pipetting can further reduce variability by eliminating repetitive manual operations. Pipetting two aliquots of a sample into adjacent wells is far less likely to cause assay variability when compared with more complex operations such as independent sample preparations in separate runs. This probably explains why intra-assay variation is typically much tighter than inter-assay variation in most LBAs. Second, with the advancement in science and technology, novel highly reproducible platforms have emerged that are extensively employed for bioanalytical labs. Examples of these instrumentation platforms include Meso Scale Diagnostic ${ }^{T M}{ }^{\circledR S D}{ }^{\circledR}$ (MesoScale Discovery) (electro-chemiluminescent immunoassay), Gyros Protein Technologies ${ }^{T M}$ Gyrolab $^{\circledR}$ (disc-based microfluidic system), Singulex ${ }^{T M}$ Erenna ${ }^{\circledR}$ and Quanterix ${ }^{T M}$ Simoa ${ }^{\circledR}$ (paramagnetic bead based systems) and Protein Simple ${ }^{T M}$ ELLA Simple Plex ${ }^{\circledR}$ (cartridge-based microfluid system). LBA methods using such automation, multiplexing and multiarray systems are providing excellent precision and accuracy, high-throughput and ultimate sensitivity with reduced costs. Last, the utilization of high-quality reagents, optimized method designs and automatic liquid handlers has also improved assay performance to a significant extent. 
Current regulatory guidelines specifying replicate analysis in LBA method validation differ. The US FDA [3] mentions that accuracy and precision can be improved by the use of duplicate analysis but only recommends duplicate analysis for calibrator samples and quality control samples, whereas the European Medicine Agency guideline [4] recommends the analysis of calibrators, quality control and study samples at least in duplicate. Does duplicate analysis necessarily correlate with improved precision and accuracy? Can singlicate analysis deliver the same quality of data as duplicate analysis? The 6th Workshop on Recent Issues in Bioanalysis (WRIB) meeting in 2012 addressed this topic and reached a consensus: "It is generally accepted to run singlicate analysis once the assay has been demonstrated to be robust" [5]. At the Crystal City V meeting in 2013, a similar consensus was reached: "The concept of running singlet analysis for LBAs if the method allows this and the number of replicates can be driven by the data during validation" [6]. The 10th European Bioanalysis Forum (EBF) Workshop in Barcelona, November 2017, opened a panel discussion solely on this topic, and each panelist (Enric Bertran of Roche Innovation Center Basel, Johannes Stanta of Covance, Craig Stovold of AstraZeneca and James Lawrence of Envigo) presented comparison studies of duplicate analysis results with simultaneous singlet evaluation of designated duplicates. Their data demonstrated that the outcome of key parameters both for validation and study samples was within a one-digit difference between duplicate and singlicate analysis. Matthew Barfield (from GSK) mentioned that GSK had filed five successful submissions of compounds, all done in singlicate analysis, without raising any concerns from regulatory authorities (during workshop WS-8: Single vs Duplicates, EBF 10th Open Symposium 15-17 November 2017). A few groups already have taken the initiative to publish their work comparing duplicate and singlicate analysis for regulated PK LBA performance. At the 7th EBF workshop in 2014, the QPS (Quest Pharmeceutical Services, LLC) Netherlands LBA group recalculated the results using singlicate approaches out of duplicate results. Their data showed that there were no significant differences between prestudy validation, in-study validation including incurred sample reanalysis (ISR), and test sample results between duplicates and singlicates [7]. Clark and his colleagues evaluated the relative precision of duplicates compared with singlicates using 60 Gyrolab datasets containing over 23,000 replicate pairs in up to 23 assays. They found no statistically significant bias in intrasubject variability between the replicates, while a significant intersubject variability was observed. They concluded that running replicates from the same sample will not significantly reduce variation or change PK parameters [8]. Hottenstein et al. also compared a GSK ligand-binding method validation performed in singlicate for a biotherapeutic fusion protein with the results from QPS where the validation was completed in duplicate. They concluded that LBAs performed by singlicate analysis can deliver robust data as duplicate analysis, which meets current regulatory and industry guidance [9].

\section{Singlicate analysis: regulatory acceptance $\&$ scientific implementation}

Although the acceptance of singlicate analysis has been proposed for a while, the regulated bioanalytical community remains apprehensive of accepting singlicate analysis into routine practice. What is preventing them from doing so? The reason is seemingly due to the absence of a clear regulatory guideline for singlicate analysis. Unless sufficient evidence is collected, regulatory authorities may not be convinced that the singlicate analysis approach is sound. The burden-of-proof to generate supporting data for singlicate analysis is with pharmaceutical companies and CROs. To shift the industry paradigm, more solid data demonstrating comparable precision and accuracy results between singlicate and duplicate analysis are needed.

In fact, singlicate analysis is not just wishful thinking. Singlicate analysis is standard practice employed in clinical diagnostic labs. Some may argue that this is mainly due to the application of automatic analyzers and the requirements to adhere to CAP/CLIA/CLSI regulations in validating and applying rugged assays to support patient diagnosis and medical treatment decisions. Singlicate analysis has also been the standard in regulated LC-MS labs for decades. Some may argue that this is because LC-MS assays incorporate an internal standard. Jo Goodman (from MedImmune) at the 10th EBF open symposium pointed out that the internal standard used in LC-MS assays is to normalize the data and compensate for potential sample lost during extraction, injection and other pretreatment processes [PERS. COMM.]. However, newer LC-MS assays being applied to biotherapeutics (e.g., hybrid LBA-LC-MS and SISCAPA techniques) are multistep methods that usually are more complex than LBA assays. Considering these examples, implementation of singlicate analysis in LBA-like LC-MS methods seems reasonable to accept. 


\section{Singlicate analysis in biomarker LBAs: future perspective \& conclusion}

So far, data from publications and conferences have indicated that singlicate analysis could be the future direction for regulated LBAs. In our opinion, singlicate analysis in nonregulated biomarker bioanalysis easily could be adopted as standard practice. Conceptually, fit-for-purpose biomarker assay validation is intended to be a flexible, dynamic model for assay characterization. Biomarker assays provide evidentiary data intended to support its context of use, in other words, the stage of development of the drug candidate. We propose adopting a standard of singlicate analysis for fit-for-purpose biomarker LBAs if it can be concluded from validation data that singlicate analysis is robust. Scientific judgment should be made to determine if duplicate or singlicate analysis is more appropriate when an assay is tricky or not robust. If the biomarker data are for critical decision-making and drug submission, adopting a 'regulated PK assay model' of duplicate analysis with tightened QC acceptance criteria is recommended. Previous method validation or sample analysis data conducted with duplicate analysis also can be re-evaluated to assess robustness using singlicate analysis. It is anticipated that no statistically significant difference between singlicate and duplicate analysis would be found. Another alternative, although not common, ISR can be conducted. ISR analysis provides valuable in-study validation data to further support assay reproducibility. One example is the Protein Simple ${ }^{\circledR}$ ELLA system, which yields triplicate results from a single well was used for an exploratory sHer-2 biomarker assay in our lab and $97 \%$ of the 300 ISR results compared within 30\% to the original result [10].

Birnboeck et al. stated, "We are convinced that singlicate analysis can become a next evolutionary step in the conduct of LBAs, especially in combination with automation" [11]. We strongly believe that adopting singlicate bioanalysis in biomarkers will not only reduce the financial burden of drug development, but also pave the way to industry-wide acceptance of singlicate analysis for both regulated and nonregulated LBAs.

\section{Acknowledgements}

The authors thank $R$ Jenkins for his critical review of this manuscript.

\section{Financial \& competing interests disclosure}

The authors have no relevant affiliations or financial involvement with any organization or entity with a financial interest in or financial conflict with the subject matter or materials discussed in the manuscript. This includes employment, consultancies, honoraria, stock ownership or options, expert testimony, grants or patents received or pending, or royalties.

No writing assistance was utilized in the production of this manuscript

\section{Open access}

This work is licensed under the Attribution-NonCommercial-NoDerivatives 4.0 Unported License. To view a copy of this license, visit http://creativecommons.org/licenses/by-nc-nd/4.0/

\section{References}

1. Lee JW, Devanarayan V, Barrett YC et al. Fit-for-purpose method development and validation for successful biomarker measurement. Pharm. Res. 23(2), 312-328 (2006).

2. Jing T, Patrick B. Parallelism experiments to evaluate matrix effects, selectivity and sensitivity in ligandbinding assay method development: pros and cons. Bioanalysis 4(18), 2213-2226 (2017).

3. US FDA. Draft Guidance for Industry: Bioanalytical Method Validation. www.fda.gov/downloads/drugs/guidances/ucm368107.pdf

4. European Medicines Agency. Guideline on Bioanalytical Method Validation. EMA/CHMP/EWP/192217/2009, 21 (2011). http: //www.ema.europa.eu/docs/en_GB/document_library/Scientific_guideline/2011/08/WC500109686.pdf

5. DeSilva B, Garofolo F, Rocci M et al. 2012 White paper on recent issues in bioanalysis and alignment of multiple guidelines. Bioanalysis 4(18), 2213-2226 (2012).

6. Booth B, Arnold ME, DeSilva B et al. Workshop report: crystal city V-quantitative bioanalytical method validation and implementation: the 2013 revised FDA guidance. AAPS J. 17(2), 277-288 (2015).

7. QPS Holdings, LLC. Conducting ELISAs: are duplicates always necessary or do singlicates suffice? www.qps.com/news-boianalysis7--2014.php

8. Clark TH, Yates PD, Chunyk AG et al. Feasibility of singlet analysis for ligand-binding assays: a retrospective examination of data generated using the gyrolab platform. AAPS J. 18(5), 1300-1308 (2016).

9. Hottenstein CS, Becker C, Asher C et al. Case study analysis of singlicate versus duplicate ligand-binding assay performance of a single bioanalytical method. AAPS Meeting Poster Abstract, NBC 2015 collection. http://abstracts.aaps.org/Verify/NBC15/PosterSubmissions/M1056.pdf 
10. Zhuqiu Y, Tu J, James H et al. Simple PlexTM ELLA: a high throughput microfluidic immunoassay platform for the detection of human sHER-2 in human serum. Poster presented at: EBF 10th Open Symposium,15-17 November 2017.

11. Birnboeck HF, Schick E, Justies N. Singlicate analysis in regulated bioanalysis using ligand-binding assays: where are we heading? Bioanalysis 9(17), 1357-1359 (2017). 